\title{
Four-level and two-qubit systems, sub-algebras, and unitary integration
}

\author{
A. R. P. Rau ${ }^{1, *}$, G. Selvaraj ${ }^{1}$ and D. Uskov ${ }^{1,2}$ \\ ${ }^{1}$ Department of Physics and Astronomy, Louisiana State University, Baton Rouge, Louisiana 70803-4001, USA. \\ 2 Department of Optics, P.N.Lebedev Physicsl Institute, 119991 Moscow, Russia.
}

\begin{abstract}
Four-level systems in quantum optics, and for representing two qubits in quantum computing, are difficult to solve for general time-dependent Hamiltonians. A systematic procedure is presented which combines analytical handling of the algebraic operator aspects with simple solutions of classical, first-order differential equations. In particular, by exploiting $s u(2) \oplus s u(2)$ and $s u(2) \oplus s u(2) \oplus u(1)$ sub-algebras of the full $S U(4)$ dynamical group of the system, the non-trivial part of the final calculation is reduced to a single Riccati (first order, quadratically nonlinear) equation, itself simply solved. Examples are provided of two-qubit problems from the recent literature, including implementation of two-qubit gates with Josephson junctions.
\end{abstract}

PACS numbers: 03.65.Yz, 05.30.-d, 42.50.Lc

Four-level systems are often of interest in quantum optics and in multiphoton processes. As examples of recent literature, see, for instance, 1, 2]. Added interest is provided by recent applications for quantum information and computing where two qubits constitute a four-level system. Indeed, two qubits and associated logic gates have been practically realized through Josephson junctions [3], the coupling Hamiltonian viewed as a $4 \times 4$ matrix.

In recent papers, we have developed a "unitary integration" technique for solving time-dependent operator equations such as the Schrödinger or Liouville equation for the state or density matrix of a quantum system [4]. The method also extends to more general master equations when dissipation and decoherence are present 5 . The evolution operator is written in the form of a product of exponentials, each exponent itself a product of a classical function of time and one quantum operator. When these operators form a closed algebra under commutations, first-order time-dependent equations can be derived for the introduced classical functions, and their resulting solutions allow the construction of a complete solution of the quantal problem. The method works best, of course, when the number of operators in the algebra is small. For one qubit, where the most general Hamiltonian involves only four operators, the three Pauli matrices and the unit matrix, the $s u(2)$ algebra has just three elements. Upon including dissipation and decoherence, a $3 \times 3$ matrix for the elements of the density matrix introduces, at its most general, eight elements of an $s u(3)$ algebra [5]. For special situations when an $s u(2)$ subalgebra suffices, such decoherence problems can also be treated through just three classical functions whose defining equations further reduce to just one non-trivial Riccati (first-order, quadratically nonlinear) equation (or, equivalently, to a linear second-order differential equation).

The aim of this paper is to examine two-qubit or fourlevel problems analogously. The complete description in terms of the 15 operators of the symmetry group $S U(4)$ (together with the unit operator) requires 15 exponential factors in the evolution operator which is cumbersome. However, judicious choices of sub-algebras reduce the problems considerably. These may be of interest since they describe several of the two-qubit logic gates and four-level systems in the recent literature. Specifically, we present here the cases pertaining to two sub-algebras, $s u(2) \oplus s u(2)$ and $s u(2) \oplus s u(2) \oplus u(1)$. In both instances, the same single Riccati equation for a single function is all that remains to be solved (through, for example, a popular Mathematica package [ $[$ ]) to provide complete descriptions of such systems. Other sub-algebras, namely $s o(5)$ with ten operators and $s u(3)$ with eight, will be the subject of future work.

\section{ALGEBRA OF OPERATORS FOR A FOUR-STATE SYSTEM}

$$
S U(2) \times S U(2) \text { sub-groups }
$$

The sixteen linearly independent operators of a fourstate system can be chosen in a variety of matrix representations. One choice, familiar in nuclear and particle physics applications [7], are the $15 \lambda_{i}$ matrices of $s u(4)$. This set is suitable for examining $s u(3)$ and $s u(2)$ subalgebras involving 8 and 3 operators, respectively, but for our interest in this paper, an alternative representation proves more convenient. This starts from two qubits and employs their individual Pauli matrices, 6 in number, along with tensor products, 9 in number: $\frac{1}{2} \vec{\sigma}, \frac{1}{2} \vec{\tau}, \frac{1}{2} \vec{\sigma} \otimes \frac{1}{2} \vec{\tau}$. Together with the unit $4 \times 4$ matrix, these 16 matrices called $O_{i}$ are explicitly tabulated in an earlier paper [8]. (A slightly different arrangement of the same operators, called $X_{i}$, with much useful group-theoretic discussion, is in $[9]$ ). They have the advantage for the unitary integration procedure (to be described below) that the square of each is, to within a factor, the unit matrix. Exponentiation of these operators is thereby rendered simple, involving just two terms. 


\begin{tabular}{|c|c|c|c|c|c|c|c|c|c|c|c|c|c|c|c|}
\hline$O_{X}$ & $\mathrm{O}_{2}$ & $\mathrm{O}_{3}$ & $\mathrm{O}_{4}$ & $O_{5}$ & $\mathrm{O}_{6}$ & $\mathrm{O}_{7}$ & $O_{8}$ & $O_{9}$ & $O_{10}$ & $O_{11}$ & $O_{12}$ & $O_{13}$ & $O_{14}$ & $O_{15}$ & $O_{16}$ \\
\hline $\mathrm{O}_{2}$ & 0 & 0 & 0 & $i O_{6}$ & $-i O_{5}$ & $i O_{8}$ & $-i O_{7}$ & 0 & 0 & 0 & 0 & $i O_{16}$ & $-i O_{15}$ & $i O_{14}$ & $-i O_{13}$ \\
\hline$O_{3}$ & 0 & 0 & 0 & 0 & 0 & 0 & 0 & $i O_{10}$ & $-i O_{9}$ & $i O_{12}$ & $-i O_{11}$ & $i O_{15}$ & $-i O_{16}$ & $-i O_{13}$ & $i O_{14}$ \\
\hline $\mathrm{O}_{4}$ & 0 & 0 & 0 & $i O_{8}$ & $-i O_{7}$ & $\frac{i}{4} O_{6}$ & $\frac{-i}{4} O_{5}$ & $i O_{12}$ & $-i O_{11}$ & $\frac{i}{4} O_{10}$ & $-\frac{i}{4} O_{9}$ & $\begin{array}{l}0 \\
\end{array}$ & 0 & 0 & 0 \\
\hline$O_{5}$ & $-i O_{6}$ & $\overline{0}$ & $-i O_{8}$ & 0 & $i \mathrm{O}_{2}$ & 0 & $i O_{4}$ & $\begin{array}{l}0 \\
\end{array}$ & 0 & $-i O_{16}$ & $-i O_{14}$ & 0 & $i O_{12}$ & 0 & $i O_{11}$ \\
\hline$\overline{O_{6}}$ & $i O_{5}$ & 0 & $i O_{7}$ & $-i O_{2}$ & 0 & 0 & $-i O_{4}$ & 0 & $\overline{0}$ & 0 & $i O_{15}$ & $-i O_{11}$ & 0 & $-i O_{12}$ & 0 \\
\hline$O_{7}$ & $-i O_{8}$ & 0 & $\frac{-i}{4} O_{6}$ & 0 & $i O_{4}$ & 0 & $\frac{i}{4} O_{2}$ & $i O_{15}$ & $-i O_{13}$ & 0 & 0 & $\frac{i}{4} O_{10}$ & 0 & $\frac{-i}{4} O_{9}$ & 0 \\
\hline$O_{8}$ & $i O_{7}$ & 0 & $\frac{i}{4} O_{5}$ & $-i O_{4}$ & 0 & $\frac{-i}{4} O_{2}$ & 0 & $i O_{14}$ & $-i O_{16}$ & 0 & 0 & 0 & $\frac{-i}{4} O_{9}$ & 0 & $\frac{i}{4} O_{10}$ \\
\hline$O_{9}$ & 0 & $-i O_{10}$ & $-i O_{12}$ & 0 & 0 & $-i O_{15}$ & $-i O_{14}$ & 0 & $i O_{3}$ & $\overline{0}$ & $i O_{4}$ & 0 & $i O_{8}$ & $i O_{7}$ & 0 \\
\hline$O_{10}$ & 0 & $i O_{9}$ & $i O_{11}$ & 0 & 0 & $i O_{13}$ & $i O_{16}$ & $-i O_{3}$ & 0 & $-i 0_{4}$ & 0 & $-i O_{7}$ & 0 & 0 & $-i O_{8}$ \\
\hline$O_{11}$ & 0 & $-i O_{12}$ & $\frac{-i}{4} O_{10}$ & $i O_{16}$ & $-i O_{13}$ & 0 & 0 & 0 & $i O_{4}$ & 0 & $\frac{i}{4} O_{3}$ & $\frac{i}{4} O_{6}$ & 0 & 0 & $\frac{-i}{4} O_{5}$ \\
\hline$O_{12}$ & 0 & $i O_{11}$ & $\frac{i}{4} O_{9}$ & $i O_{14}$ & $-i O_{15}$ & 0 & 0 & $-i O_{4}$ & 0 & $\frac{-i}{4} O_{3}$ & 0 & 0 & $\frac{-i}{4} O_{5}$ & $\frac{i}{4} O_{6}$ & 0 \\
\hline$O_{13}$ & $-i O_{16}$ & $-i O_{15}$ & 0 & 0 & $i O_{11}$ & $\frac{-i}{4} O_{10}$ & 0 & $\begin{array}{l}0 \\
\end{array}$ & $\mathrm{iO}_{7}$ & $\frac{-i}{4} O_{6}$ & 0 & $\overline{0}$ & 0 & $\frac{i}{4} O_{3}$ & $\frac{i}{4} O_{2}$ \\
\hline$O_{14}$ & $i O_{15}$ & $i O_{16}$ & $\overline{0}$ & $-i O_{12}$ & 0 & 0 & $\frac{2}{4} O_{9}$ & $-i O_{8}$ & 0 & 0 & $\frac{\imath}{4} O_{5}$ & 0 & 0 & $\frac{-2}{4} \frac{-2}{4} O_{2}$ & $\frac{-2}{4} O_{3}$ \\
\hline$O_{15}$ & $-i O_{14}$ & $i O_{13}$ & 0 & 0 & $i 0_{12}$ & $\frac{i}{4} O_{9}$ & 0 & $-i O_{7}$ & 0 & 0 & $\frac{-i}{4} O_{6}$ & $\frac{-i}{4} O_{3}$ & $\frac{i}{4} O_{2}$ & 0 & 0 \\
\hline$O_{16}$ & $i O_{13}$ & $-i O_{14}$ & 0 & $-i O_{11}$ & 0 & 0 & $\frac{-i}{4} O_{10}$ & 0 & $i O_{8}$ & $\frac{i}{4} O_{5}$ & 0 & $\frac{-i}{4} O_{2}$ & $\frac{i}{4} O_{3}$ & 0 & 0 \\
\hline
\end{tabular}

TABLE I: Table of commutators. With operators $O_{i}$ in the first column and $O_{j}$ in the top row, each entry provides the commutator $\left[O_{i}, O_{j}\right]$.

Table I gives the commutators $\left[O_{i}, O_{j}\right]$ and is useful for picking out sub-algebras. For example, $\left[\mathrm{O}_{2}, \mathrm{O}_{5}\right]=i \mathrm{O}_{6}$. The four states in this representation may be described either in the four-state language as the column vectors $(1,0,0,0),(0,1,0,0),(0,0,1,0),(0,0,0,1)$, or in spin notation as $|\uparrow \uparrow\rangle,|\uparrow \downarrow\rangle,|\downarrow \uparrow\rangle,|\downarrow \downarrow\rangle$, respectively.

There is a large set of six-dimensional sub-algebras formed out of two triplets obeying $s u(2)$ or, equivalently, so(3) angular momentum commutation relations. One such set, $\left(\mathrm{O}_{2}, \mathrm{O}_{5}, \mathrm{O}_{6} ; \mathrm{O}_{3}, \mathrm{O}_{9}, \mathrm{O}_{10}\right)$, is the trivial $s u(2) \oplus s u(2)$ subalgebra of local operations on two qubits $\vec{\sigma}$ and $\vec{\tau}$. There are many equivalent sets generated by a Similarity Transformation (ST) acting on the elements of a subalgebra by right-left multiplication by an arbitrary element of the full $S U$ (4) group. Let $W$ be a unitary $4 \times 4$ matrix, and $x$ an su(4) algebra element. Then, ST acts as

$$
x^{\prime}=W x W^{-1} \equiv W x W^{\dagger} .
$$

In this section we will use only a discrete subgroup of all possible ST transformations, which have the property that the set of 15 operators $O_{i}$ is transformed into the same set $O_{j}$, i.e. the action of $\mathrm{ST}$ is equivalent to a permutation among the $O_{i}$. In this case the $s u(2) \oplus s u(2)$ subalgebras, obtained by ST transformation of $\vec{\sigma}, \vec{\tau}$ can be identified by direct analysis of subgroup structures using Table 1.

Subgroups different from the trivial $\vec{\sigma}, \vec{\tau}$ one can be generated only by two-qubit entangling operations, such as transformation to the Bell basis (or magic Bell basis [10]). One can easily verify that the set $\left(\mathrm{O}_{9}, \mathrm{O}_{8}, \mathrm{O}_{14} ; \mathrm{O}_{6}, \mathrm{O}_{11}, \mathrm{O}_{13}\right)$ is such a subalgebra. That is, the operator sets $\left(\tau_{x}, \sigma_{y} \tau_{z}, \sigma_{y} \tau_{y}\right)$ and $\left(\sigma_{y}, \sigma_{z} \tau_{x}, \sigma_{x} \tau_{x}\right)$ are two mutually commuting triplets with $s u(2)$ commutation relations. The ST transformation with the unitary matrix

$$
\frac{1}{\sqrt{2}}\left(\begin{array}{cccc}
1 & 0 & 0 & 1 \\
0 & 1 & 1 & 0 \\
0 & 1 & -1 & 0 \\
1 & 0 & 0 & -1
\end{array}\right)
$$

passes from the $\vec{\sigma}$ set to $\left(\tau_{x}, \sigma_{y} \tau_{z}, \sigma_{y} \tau_{y}\right)$ and from $\vec{\tau}$ to $\left(\sigma_{y}, \sigma_{z} \tau_{x}, \sigma_{x} \tau_{x}\right)$. The basis corresponding to Eq. (11) is the Bell basis, $(1,0,0,1)=|\uparrow \uparrow\rangle+|\downarrow \downarrow\rangle,(0,1,1,0)=$ $|\uparrow \downarrow\rangle+|\downarrow \uparrow\rangle,(0,1,-1,0)=|\uparrow \downarrow\rangle-|\downarrow \uparrow\rangle,(1,0,0,-1)=$ $|\uparrow \uparrow\rangle-|\downarrow \downarrow\rangle$, which are simultaneous eigenstates of $\sigma_{x} \tau_{x}$ and $\sigma_{y} \tau_{y}[10]$.

All such subalgebras are maximal $s u(2) \oplus s u(2)$ subalgebras of $s u(4)$, in the sense that, if any operator from Table I is added to the six already chosen, the algebra closes (as a Lie algebra) only in the full $s u(4)$ algebra. Another perspective on the above Bell basis is provided by considering a four-level system with nearest neighbor couplings as in the Hamiltonian,

$$
H=\left(\begin{array}{llll}
0 & \alpha & 0 & 0 \\
\alpha & 0 & \beta & 0 \\
0 & \beta & 0 & \gamma \\
0 & 0 & \gamma & 0
\end{array}\right)
$$

Such a matrix may be viewed as built up of three symmetric $4 \times 4$ matrices $m_{j k}$, with unit entries in row (column) $j$ and column(row) $k$ and zero everywhere else, so that $H=\alpha m_{12}+\beta m_{23}+\gamma m_{34}$. Starting with these three real, symmetric matrices, we define two non-zero commutators between them, $m_{13}=-i\left[m_{12}, m_{23}\right], m_{24}=$ $-i\left[m_{23}, m_{34}\right]$ to give two imaginary, anti-symmetric matrices, and a final real symmetric $m_{14}=-i\left[m_{13}, m_{34}\right]$ to complete a full 6-dimensional so(4) algebra. Using the well-known relation between $S O(4)$ and $S U(2) \times$ 
$S U(2)$ groups, this sub-algebra decomposes into two sets of mutually commuting triplets, $m_{12}+m_{34}, m_{13}-$ $m_{24}, m_{12}-m_{34}$ and $m_{12}-m_{34}, m_{13}+m_{24}, m_{23}-m_{14}$. These linear combinations correspond to the subalgebra $\left(\mathrm{O}_{9}, \mathrm{O}_{8}, \mathrm{O}_{14} ; \mathrm{O}_{6}, \mathrm{O}_{11}, \mathrm{O}_{13}\right)$ of the full $s u(4)$ algebra. Such a construction generalizes to $n$-level systems, $n>4$, with nearest neighbor couplings. The matrices $m_{j k}$ defined as

$$
\left(m_{j k}\right)_{p q}=(-i)^{|j-k|-1}\left[\delta_{j p} \delta_{k q}+(-1)^{k-j-1} \delta_{j q} \delta_{k p}\right]
$$

provide $n(n-1) / 2$ generators of a $s o(n)$ algebra. These form a sub-algebra of the $n^{2}-1$-dimensional $s u(n)$ algebra although, for $n>4$, the $s o(n)$ no longer reduces to a product of $s u(2)$ algebras.

$$
S U(2) \times S U(2) \times U(1) \text { sub-groups }
$$

A class of maximal seven-dimensional sub-algebras of $s u(4)$, different from the one considered in the previous subsection, can be constructed by taking linear combinations of $O_{n}$ generators. Inspection of Table I shows that the first seven elements form a diagonal sub-block closed under commutation, isomorphic to a so(4) algebra. With $\mathrm{O}_{3}$ commuting with all the rest of this sub-block, the other six divide into two mutually commuting $s u(2)$ triplets upon forming appropriate linear combinations which pass from a standard $4 \times 4$ antisymmetric real-matrix representation of $s o(4)$ to $s u(2) \oplus s u(2)$ matrix $(4 \times 4)$ form. Another such example, previously considered by one of us [8], is provided by $\left(\mathrm{O}_{2}, \mathrm{O}_{3}, \mathrm{O}_{13}, \mathrm{O}_{14}, \mathrm{O}_{15}, \mathrm{O}_{16}\right)$, with $\mathrm{O}_{4}$ commuting with all of them. The linear combinations, defined in [8] as "pseudo-spins", obey $s u(2)$ commutation relations while not being isomorphic to Pauli spinors. While the Pauli matrices constitute a matrix algebra with the unit operator as the identity element, the pseudo-spin matrix algebra has as its identity element the operator $\frac{1}{2}\left(\hat{I} \pm \sigma_{z} \tau_{z}\right)$. One implication is that this particular type of $s u(2) \oplus s u(2)$ algebra is not related by a ST operation to the $s u(2) \oplus s u(2)$ algebras considered in the previous section. These $s u(2) \oplus s u(2) \oplus u(1)$ sets arise, for example, in the physical context of nuclear magnetic resonance [8] with scalar coupling and heteronuclear twospin coherences. For applications in methods such as unitary integration which require closure under commutation, such pseudo-spins work equally well as we will see below. There are many such sub-algebras which can be obtained by the discrete subgroup of ST operations as discussed in the previous section. For example, one can simply relabel $(x, y, z)$. Indeed, each row (or column) of Table I has seven zero entries. The corresponding seven operators constitute such an algebra for a total of 15 sets 11.
The recent demonstration of two qubit gates formed out of Josephson junctions [3] considers the Hamiltonian,

$$
H=\left(\begin{array}{cccc}
E_{00} & -\frac{1}{2} E_{J 1} & -\frac{1}{2} E_{J 2} & 0 \\
-\frac{1}{2} E_{J 1} & E_{10} & 0 & -\frac{1}{2} E_{J 2} \\
-\frac{1}{2} E_{J 2} & 0 & E_{10} & -\frac{1}{2} E_{J 1} \\
0 & -\frac{1}{2} E_{J 2} & -\frac{1}{2} E_{J 1} & E_{00}
\end{array}\right)
$$

Expressing in terms of the $O_{i}$ matrices in Table I, this Hamiltonian is a linear combination of $\left(\mathrm{O}_{1}, \mathrm{O}_{4}, \mathrm{O}_{5}, \mathrm{O}_{9}\right)$. Together with $\left(\mathrm{O}_{8}, \mathrm{O}_{12}, \mathrm{O}_{14}\right)$, they form a closed subalgebra, and the operator $O_{13}$ commutes with all of them. For applications below in Section 2, we record the Hamiltonian in terms of the Pauli spinors,

$H=\frac{1}{2}\left(E_{00}+E_{10}\right)-\frac{1}{2}\left(E_{J 2} \sigma_{x}+E_{J 1} \tau_{x}\right)+\frac{1}{2}\left(E_{00}-E_{10}\right) \sigma_{z} \tau_{z}$,

and

$$
\begin{aligned}
H & =\frac{1}{2}\left(E_{00}+E_{10}\right) \mathcal{I}+\frac{1}{2}\left(\omega_{+} S_{z}+\omega_{-} s_{z}\right) \\
& +\frac{1}{4}\left(E_{00}-E_{10}\right)\left(s_{+}+s_{-}-S_{+}-S_{-}\right),
\end{aligned}
$$

where we have defined $\omega_{ \pm}=-E_{J 2} \mp E_{J 1}, S_{z}=\frac{1}{2}\left(\sigma_{x}+\right.$ $\left.\tau_{x}\right), s_{z}=\frac{1}{2}\left(\sigma_{x}-\tau_{x}\right)$, and

$S_{ \pm}=\frac{1}{2}\left(\sigma_{y} \pm i \sigma_{z}\right)\left(\tau_{y} \pm i \tau_{z}\right), s_{ \pm}=\frac{1}{2}\left(\sigma_{y} \pm i \sigma_{z}\right)\left(\tau_{y} \mp i \tau_{z}\right)$.

The sets $\left(S_{z}, S_{ \pm}\right)$and $\left(s_{z}, s_{ \pm}\right)$mutually commute and each obeys the commutation relations of an $s u(2)$ algebra but, as in [8], they are not completely isomorphic to the $4 \times 4$ matrix $\sigma_{i}$ or $\tau_{i}$ algebras, as discussed above. In particular, as is easily seen, the squares of $S_{z}$ and $s_{z}$ are not the unit operator but involve $\sigma_{x} \tau_{x}$ as well. While sharing the same commutation relations as the similar pseudo-spins defined in [8], the sets here differ as $4 \times 4$ matrices, being in the " $x$-representation" instead of the counterpart " $z$-representation" in 8].

\section{UNITARY INTEGRATION OF THE EVOLUTION OPERATOR}

Time-dependent quantum problems can be conveniently handled through the unitary integration procedure of [4]. Solutions of the Schrödinger equation for the quantum state are obtained through the evolution operator $U(t)$ defined by

$$
i \dot{U}(t)=H(t) U(t), \quad U(0)=\mathcal{I},
$$

where an over-dot denotes differentiation with respect to time. For more general treatment that includes 
mixed states, master equations such as the Liuoville-von Neumann-Lindblad equation [12],

$$
\begin{aligned}
i \dot{\rho} & =[H, \rho]+\frac{1}{2} i \sum_{k}\left(\left[L_{k} \rho, L_{k}^{\dagger}\right]+\left[L_{k}, \rho L_{k}^{\dagger}\right]\right) \\
& =[H, \rho]-\frac{1}{2} i \sum_{k}\left(L_{k}^{\dagger} L_{k} \rho+\rho L_{k}^{\dagger} L_{k}-2 L_{k} \rho L_{k}^{\dagger}\right),(
\end{aligned}
$$

when first cast in terms of the individual density matrix elements, take a form similar to Eq. (7), namely

$$
i \dot{\eta}(t)=\mathcal{L} \eta(t)
$$

where $\eta$ are appropriate linear combinations of matrix elements of the density matrix $\rho_{i j}$ [5], such as, for example, the ones obtained by a decomposition of $\rho_{i j}$ in the set of generators of the corresponding $S U(n)$ group [13]. We have

$$
\begin{aligned}
\eta= & {\left[\rho_{11}-\rho_{22},\left(\rho_{11}+\rho_{22}-2 \rho_{33}\right) / \sqrt{3},\right.} \\
& \left(\rho_{11}+\rho_{22}+\rho_{33}-3 \rho_{44}\right) / \sqrt{6}, \rho_{12}+\rho_{21}, \\
& i\left(\rho_{12}-\rho_{21}\right), \rho_{13}+\rho_{31}, i\left(\rho_{13}-\rho_{31}\right), \rho_{14}+\rho_{41}, \\
& i\left(\rho_{14}-\rho_{41}\right), \rho_{23}+\rho_{32}, i\left(\rho_{23}-\rho_{32}\right), \rho_{24}+\rho_{42}, \\
& \left.i\left(\rho_{24}-\rho_{42}\right), \rho_{34}+\rho_{43}, i\left(\rho_{34}-\rho_{43}\right)\right] .
\end{aligned}
$$
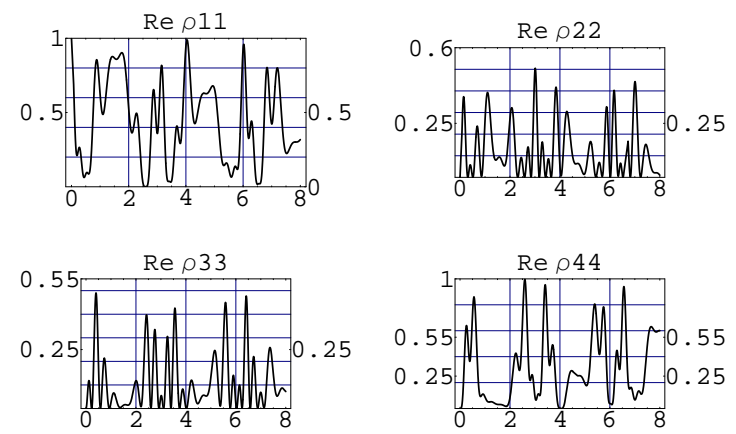

FIG. 1: Time evolution of the diagonal elements of the density matrix of a $n=4$ two-qubit Josephson junction system. The horizontal axis is $(\omega t / 2 \pi)$, parameters as given in the text with $\omega=1 \mathrm{GHz}, \delta=0$, and the initial configuration $\rho(0)=$ $\delta_{i 1} \delta_{j 1}$.

In either case of Eq. (7) or Eq. (9), unitary integration writes the solution as a product of exponentials, $\prod_{j} \exp \left[-i \mu_{j}(t) A_{j}\right]$, with time-dependent, classical functions $\mu_{j}(t)$, and $A_{j}$ the elements of a complete algebra [4]. Upon substituting this form into Eq. (7) or Eq. (9), a consistent set of defining equations for the $\mu_{j}$ follow so long as each element of the algebra is included in the above product. As shown in [4, 5], so long as one has an $s u(2)$ algebra, solving this set defining $\mu_{j}$ reduces to
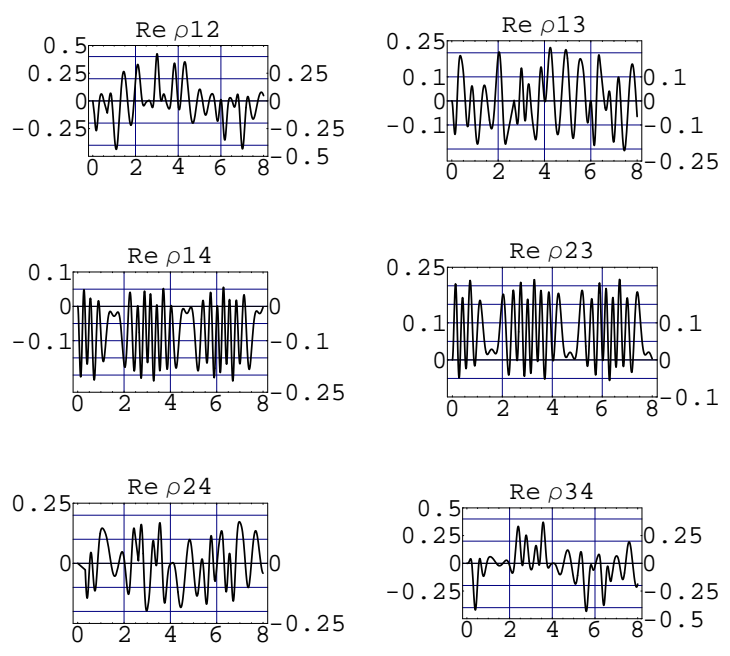

FIG. 2: As in Fig.1 but for the real part of the off-diagonal elements of the density matrix.
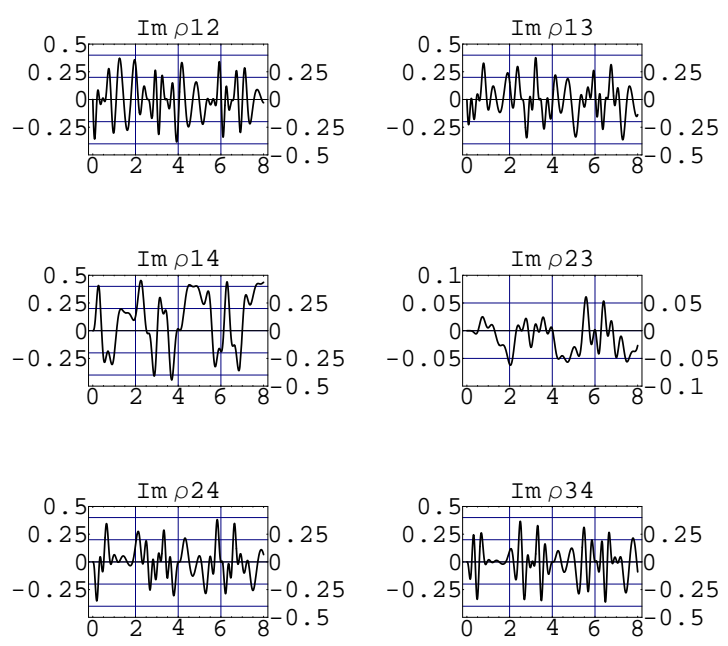

FIG. 3: As in Fig.1 but for the imaginary part of the offdiagonal elements of the density matrix.

just one non-trivial step, the solution of a single Riccati equation. This remains true if more than one $s u(2)$ is involved so long as they are mutually commuting, each set involving one Riccati equation. Note also that since only commutators of $A_{j}$ are involved in the derivation, the results apply also to pseudo-spins as in the previous section, or for other algebras such as $s o(3)$ or $s u(1,1)$ differing only in the sign or value of the structure coefficients which do not change the form of the defining equations for the $\mu$.

It is this feature that we now exploit in applying to four-level or two qubit problems that fall into the two categories of the $s u(2) \oplus s u(2)$ and $s u(2) \oplus s u(2) \oplus u(1)$ 

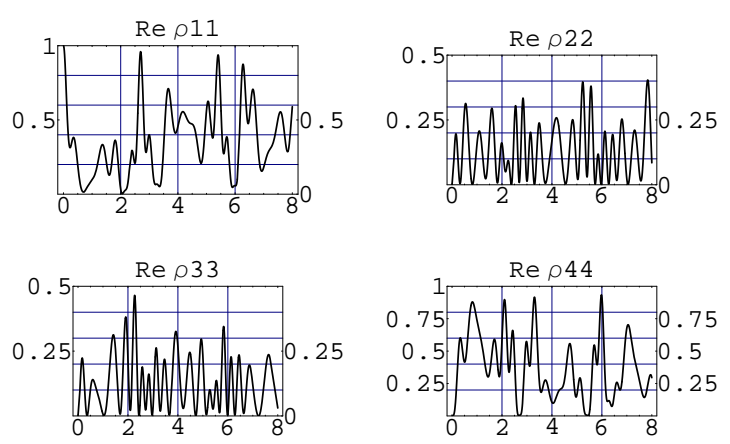

FIG. 4: Time evolution of the diagonal elements of the density matrix of a $n=4$ two-qubit Josephson junction system. The horizontal axis is $(\omega t / 2 \pi)$, parameters as given in the text with $\omega=1 \mathrm{GHz}, \delta=\pi / 4$, and the initial configuration $\rho(0)=$ $\delta_{i 1} \delta_{j 1}$. Contrast with Fig. 1 to see differences due to a mutual phase between the driving fields.
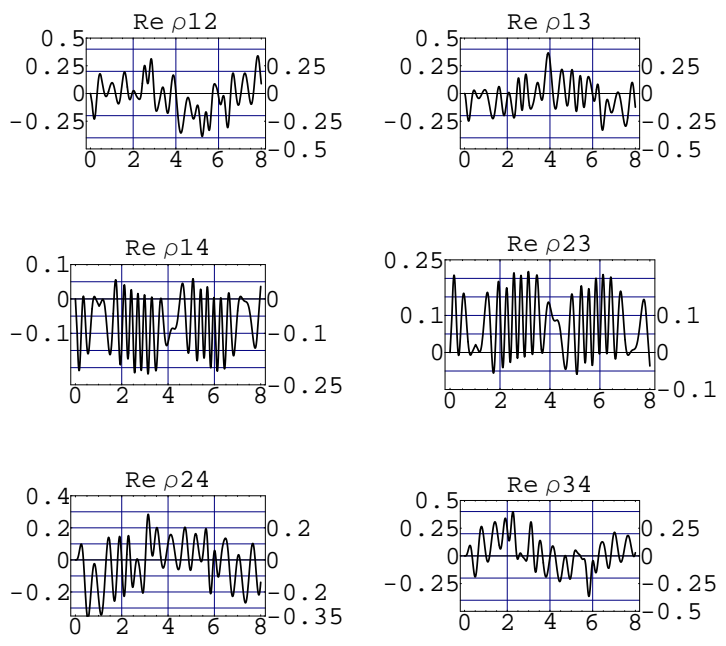

FIG. 5: As in Fig. 4 but for the real part of the off-diagonal elements of the density matrix.

sub-algebras discussed in Section 2. An example of the former is a recent discussion 14] of geometric phases with two qubits. More general time dependences than considered in that reference are amenable to our procedure presented below. For the coupled pair of Josephson junctions of [3] with the Hamiltonian in Eq. (5), we have from our previous derivation in [5] the evolution operator

$$
\begin{aligned}
U(t) & =\exp \left(-i \Omega_{0}(t)\right) \exp \left[-\frac{1}{2} i \nu_{+}(t) S_{+}\right] \exp \left[-\frac{1}{2} i \nu_{-}(t) S_{-}\right] \\
& \times \exp \left[-\frac{1}{2} i \nu_{3}(t) S_{z}\right] \exp \left[-\frac{1}{2} i \mu_{+}(t) s_{+}\right] \\
& \times \exp \left[-\frac{1}{2} i \mu_{-}(t) s_{-}\right] \exp \left[-\frac{1}{2} i \mu_{3}(t) s_{z}\right]
\end{aligned}
$$

The sets $\mu$ and $\nu$ are for the two $S U(2)$ 's involved in
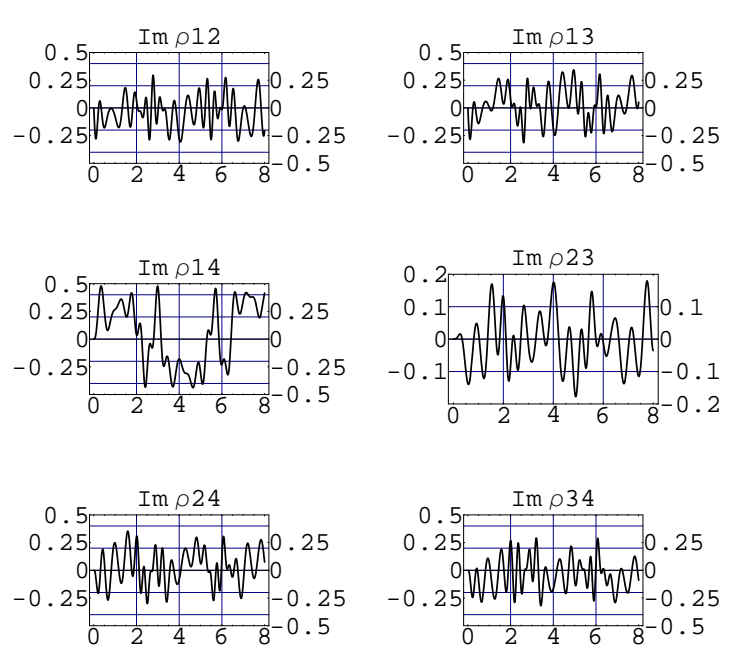

FIG. 6: As in Fig. 4 but for the imaginary part of the offdiagonal elements of the density matrix.

problems such as those in [3]. An additional term in $\sigma_{x} \tau_{x}$ in Eq. (5) could also be accommodated through an additional exponential factor in Eq. (11) and would correspond to an $\mathrm{U}(1)$. These classical functions in the exponents are obtained through the set of equations [5],

$$
\begin{aligned}
& \dot{\Omega_{0}}=\left(E_{00}+E_{10}\right) / 2, \\
& \dot{\mu_{+}}-\mu_{+}{ }^{2} k_{-}+i \omega_{-} \mu_{+}=k_{+} \text {, } \\
& \dot{\mu_{-}}-i \mu_{-} \dot{\mu_{3}}=k_{-}, \quad \dot{\mu_{3}}-2 i k_{-} \mu_{+}=\omega_{-},
\end{aligned}
$$

with a similar set for the functions $\nu$ which have $K_{ \pm}$ instead of $k_{ \pm}$. These parameters for the $H$ in Eq. (5D) take the values $K_{ \pm}=-k_{ \pm}=\left(E_{00}-E_{10}\right) / 2$. For applications with $k$ constant as in [3], and $\omega_{ \pm}$functions of time, the first, and only non-trivial, equation of the set in Eq. (12) can also be recast as [4],

$$
\dot{\theta}(t)=k-\frac{1}{2} \omega_{-}(t) \sin 2 \theta(t)
$$

with $\mu_{+} \equiv \tan (\theta+k t)$, or

$$
\ddot{\gamma}(t)+i \omega_{-}(t) \dot{\gamma}(t)+k^{2} \gamma(t)=0,
$$

with $\mu_{+} \equiv-(1 / k)(d / d t)(\ln \gamma)$. In any of these alternative forms, $\mu_{+}$(and, similarly, $\left.\nu_{+}\right)$can be solved for a given $\omega_{ \pm}$, following which the rest of the set in Eq. (12) yields to simple integration.

In Figs. 1-3, we present results for the two-qubit Josephson junction system of [3]. The parameters of that study were $E_{00}-E_{10}=7.85 \mathrm{GHz}, E_{J 1}=13.4$ and $E_{J 2}=9.1 \mathrm{GHz}$. All these parameters were held 
constant in that study but, with an eye to future applications where these energies may vary with time, we consider such applications of our general time-dependent formalism. Specifically, were the voltages to be harmonically modulated, with $E_{J 1}=13.4 \cos (\omega t+\delta)$ and $E_{J 2}=9.1 \cos (\omega t)$, solution of Eq. (12) leads to diagonal and off-diagonal components of the density matrix shown in Figs. 1-3. Starting with an initial pure state $(1,0,0,0)$, the density matrix, $\rho(t)=U \rho(0) U^{\dagger}$ is evaluated and exhibits complicated oscillatory population of the four levels (diagonal density matrix elements in Fig. 1 which, of course, always sum to unity for all $t$ ) as also coherences (off-diagonal elements in Figs. 2 and 3). Figs. 4-6 show the effect of a phase difference between the two driving fields, leading to interesting differences when compared to Figs. 1-3 where the driving fields are in phase.
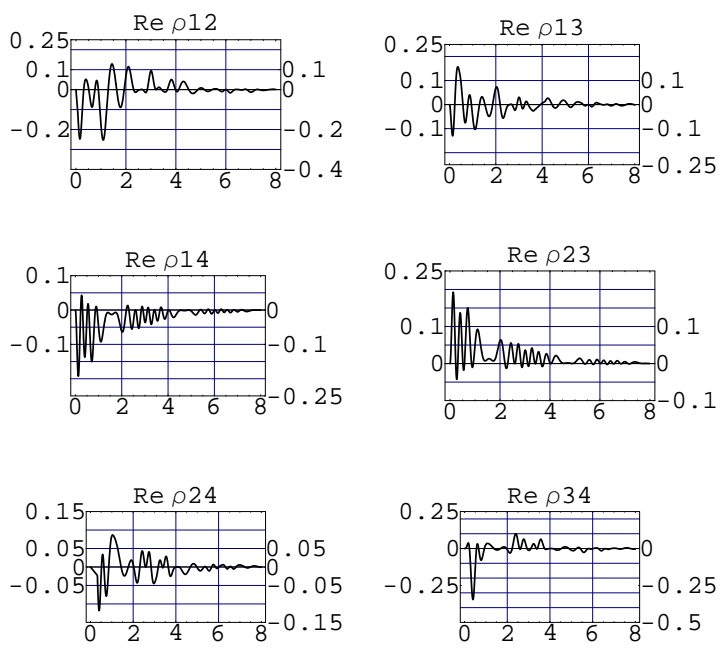

FIG. 7: As in Fig. 1 but with a damping constant $\Gamma=0.5$ $\mathrm{GHz}$. Note the differences from Fig. 1 as the oscillations damp out to give asymptotic values of $1 / 4$.

Extension to decoherence and the master equation in Eq. (8) requires handling the $15 \times 15$ problem in Eq. (9) rather than $4 \times 4$ and is necessarily more involved numerically. However, as in earlier studies [4, 5], a simplified model in which the sum in Eq. (8) runs over all $L_{k}=O_{k}$ of Table 1 reduces to the same $4 \times 4$ calculation of unitary evolution above, with the only difference being to multiply $\eta(t)$ in Eq. (10) by $\exp (-\Gamma t)$. Figs. 7-9 show the results of such a calculation for the same choice of parameters as in Figs. 1-3 but with the additional $\Gamma=0.5$ GHz. The small $t$ dependence of the two sets of figures coincide but the oscillations are damped by the decoherence such that asymptotically, the density matrix evolves to that of a mixed state, namely diagonal components of $1 / 4$ while all off-diagonal elements vanish. This evolution from a pure state to a completely mixed state is accompanied by a monotonic rise of the entropy from 0 to $\ln 4$,
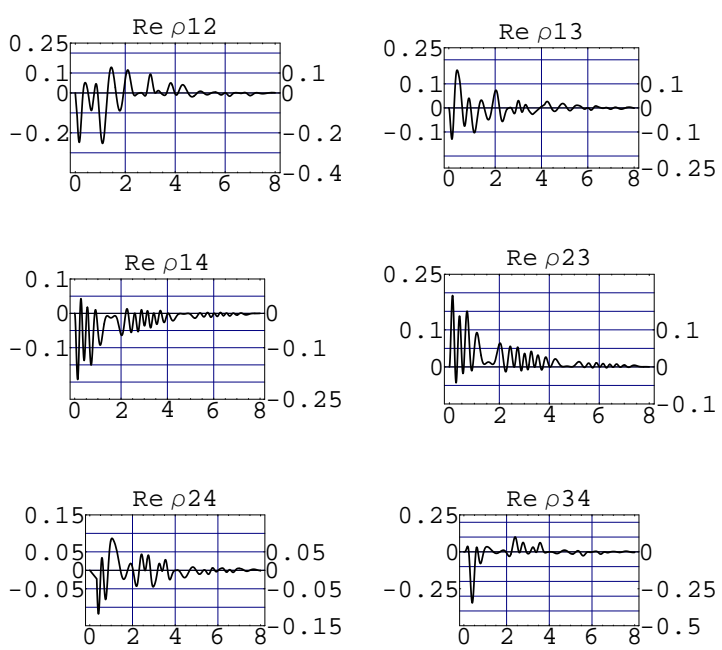

FIG. 8: As in Fig. 2 but with a damping constant $\Gamma=0.5$ GHz. Note the differences from Fig. 2 as the oscillations damp out asymptotically.
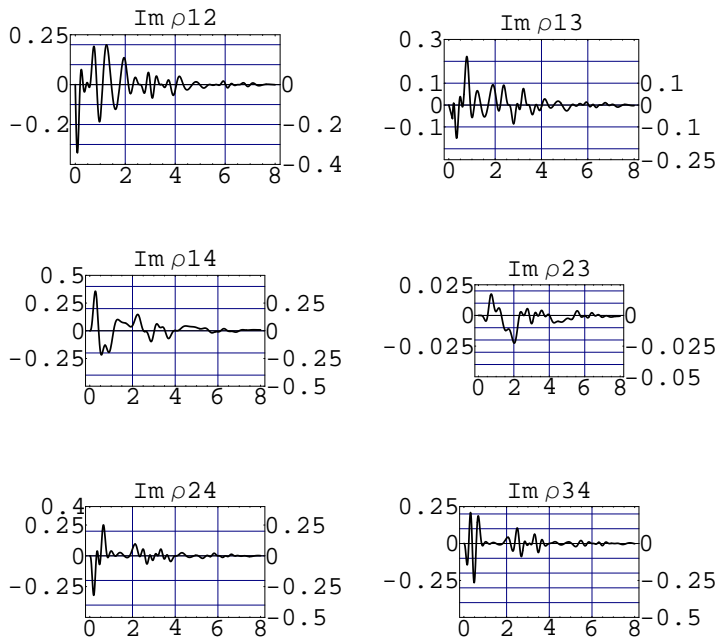

FIG. 9: As in Fig. 3 but with a damping constant $\Gamma=0.5$ $\mathrm{GHz}$. Note the differences from Fig. 3 as the oscillations damp out asymptotically.

paralleling the similar results in [4, 5].

This work has been supported by the U. S. Department of Energy and the National Science Foundation. One of us (ARPR) thanks the Tata Institute for Fundamental Research, Mumbai, India, for hospitality during the course of writing of this paper.

[*] Email: arau@phys.lsu.edu

[1] F. Legare, Phys. Rev. A 68, 063403 (2003); S. Jin, S. 
Gong, R. Li, and Z. Xu, Phys. Rev. A 69, 023408 (2004).

[2] V. Delgado and J. M. Gomez Llorente, Phys. Rev. A 68, 022503 (2003).

[3] Yu. A. Pashkin, T. Yamamoto, O. Astafiev, Y. Nakamura, D. V. Averin, and J. S. Tsai, Nature 421, 823 (2003); T. Yamamoto, Yu. A. Pashkin, O. Astafiev, Y. Nakamura, and J. S. Tsai, Nature 425, 941 (2003).

[4] A. R. P. Rau, Phys. Rev. Lett. 81, 4785 (1998). See also, J. Wei and E. Norman, J. Math. Phys. 4, 575 (1963); G. Dattoli, J. C. Gallardo, and A. Torre, Riv. Nuovo Cimento 11, No. 11, 1 (1988); G. Campolieti and B. C. Sanctuary, J. Chem. Phys. 91, 2108 (1989); B. A. Shadwick and W. F. Buell, Phys. Rev. Lett. 79, 5189 (1997).

[5] A. R. P. Rau and R. A. Wendell, Phys. Rev. Lett. 89, 220405 (2002); A. R. P. Rau and Weichang Zhao, Phys. Rev. A 68, 052102 (2003).

[6] S. Wolfram, Mathematica: A System for Doing Mathematics by Computer (Addison-Wesley, Redwood City, CA, 1988).

[7] A. W. Joshi, Elements of group theory for physicists (John Wiley, New York, 1982); W. Greiner and B. Müeller, Quantum Mechanics: Symmetries (Springer-
Verlag, Berlin, 1989).

[8] A. R. P. Rau, Phys. Rev. A 61, 032301 (2000).

[9] J. Zhang, J. Vala, S. Sastry, and K. B. Whaley, Phys. Rev. A 67, 042313 (2003).

[10] C. H. Bennett, D. P. DiVincenzo, J. Smolin, and W. K. Wootters, Phys. Rev. A 54, 3824 (1996); Scott Hill and W. K. Wootters, Phys. Rev. Lett. 78, 5022 (1997).

[11] A formal description of this procedure is that for each of the $15 O_{i}$, the set of $O_{j}$ obtained by the ST transformation, $O_{j} O_{i} O_{j}^{\dagger}=O_{i}$, provide such a $s u(2) \oplus s u(2) \oplus u(1)$ sub-algebra. Similarly, the set satisfying $O_{j} O_{i} O_{j}^{T}=-O_{i}$ provide the $s u(2) \oplus s u(2)$ or $s o(5)$ sub-algebras, respectively, for $O_{i}$ symmetric or anti-symmetric.

[12] See, for instance, D. F. Walls and G. Milburn, Quantum Optics (Springer-Verlag, Berlin, 1994); M. O. Scully and M. S. Zubairy, Quantum Optics (Cambridge University Press, Cambridge, U. K., 1996); W. P. Schleich, Quantum Optics in Phase Space (Wiley-VCH, Berlin, 2001).

[13] F. T. Hioe and J. H. Eberly, Phys. Rev. Lett. 47, 838 (1981) and Phys. Rev. A. 25, 2168 (1982).

[14] R. G. Unanyan and M. Fleischhauer, Phys. Rev. A 69, 050302(R) (2004). 